\title{
Conversion of chirp in fiber compression
}

\author{
Péter Dombi, ${ }^{1}$ Péter Rácz, ${ }^{2}$ Laszlo Veisz, ${ }^{1}$ and Peter Baum ${ }^{1,3, *}$ \\ ${ }^{1}$ Max-Planck-Institut für Quantenoptik, Hans-Kopfermann-Str. 1, 85748 Garching, Germany \\ ${ }^{2}$ Wigner Research Centre for Physics, Konkoly-Thege M. út 29-33, 1121 Budapest, Hungary \\ ${ }^{3}$ Ludwig-Maximilians-Universität München, Am Coulombwall 1, 85748 Garching, Germany \\ *Corresponding author: peter.baum@lmu.de
}

Received January 22, 2014; accepted March 2, 2014;

posted March 7, 2014 (Doc. ID 205294); published April 3, 2014

\begin{abstract}
Focusing positively chirped femtosecond pulses into nonlinear fibers provides significant spectral broadening and compression at higher pulse energies than achievable conventionally because self-focusing and damage are avoided. Here, we investigate the transfer of input to output chirp in such an arrangement. Our measurements show that the group delay dispersion of the output pulse, originating from the nonlinearities, is considerably reduced as compared to the initial value, by about a factor of 10 . The mechanism of chirp reduction is understood by an interplay of selfphase modulation with initial chirp within the fiber. A simple model calculation based on this picture yields satisfactory agreement with the observations and predicts significant chirp reduction for input pulses up to the $\mu \mathrm{J}$ regime. In practice, the reduction of chirp observed here allows for compressing the spectrally broadened intense pulses by ultrabroadband dispersive multilayer mirrors of quite moderate dispersion. (c) 2014 Optical Society of America

OCIS codes: (140.7090) Ultrafast lasers; (190.4370) Nonlinear optics, fibers; (320.1590) Chirping; (320.5520) Pulse compression.

http://dx.doi.org/10.1364/OL.39.002232
\end{abstract}

Spectral broadening and pulse compression in fibers can be extended to significantly higher pulse energies by (1) using large-mode-area photonic crystal fibers [ $[\underline{1}-\underline{6}]$ in combination with (2) chirping the input pulses $[\underline{6}, \overline{1}]$. The latter approach is particularly promising for upscaling the pulse energy because the nonlinear broadening mechanisms in the fiber scale with the pulse's peak intensity, whereas damaging effects caused by self-focusing scale with the peak power. Experiments show that chirped pulses at higher energy can provide the same amount of broadening as short pulses of lower energy [6]. Positive chirp was applied to generate $350 \mathrm{~nJ}, 16 \mathrm{fs}$ pulses at a repetition rate of $5.1 \mathrm{MHz}$ in this way [6]. Negatively chirped input can also result in some improvements [1]], although the fiber's dispersion tends to compress the propagating pulses in a nondesired way.

A central aspect of chirped broadening with practical relevance is the amount of output chirp that is generated at the high input-chirps required for making full use of the advantage of chirped broadening. Will the output chirp be low enough so that efficient compression schemes such as chirped mirrors are still practically applicable?

Here we report an experimental investigation of how the input chirp converts to an output chirp during broadening in a large-mode-area fiber. The concept of our measurement is depicted in Fig. 1. A long-cavity Ti:sapphire oscillator []] (Scientific $\overline{\mathrm{X}} \mathrm{L}$, Femtolasers $\mathrm{GmbH}$ ) provides 55 fs pulses at a central wavelength of $795 \mathrm{~nm}$ with $\sim 500 \mathrm{~nJ}$ of pulse energy at a repetition rate of 5.1 MHz. The system's internal prism compressor is used for inducing a well-defined group delay dispersion $\left(D_{\text {in }}\right)$; this stretches the pulses in time to a duration $\tau_{\text {in }}$. An aspheric lens $(f \approx 40 \mathrm{~mm})$ is used to focus the chirped pulses into a large-mode-area fiber (LMA-25, Thorlabs) for nonlinear broadening. The output beam is collimated with another lens $(f \approx 20 \mathrm{~mm})$. The output pulses have a group delay dispersion $D_{\text {out }}$ and a duration $\tau_{\text {out }}$. The output pulses were compressed with double-angled chirped mirrors [8] (details are provided below). The final pulse durations were measured by intensity autocorrelation. The fiber length was $15 \mathrm{~mm}$; shorter fibers did not yield a satisfactory performance.

The measurement proceeded as follows: starting with the shortest achievable input duration ( $55 \mathrm{fs}$ ), a pulse energy of $90 \mathrm{~nJ}$ was chosen in order to generate an output spectrum with a Fourier limit of $\sim 17 \mathrm{fs}$. This spectrum extended from $\sim 720-870 \mathrm{~nm}$. Next we introduced more and more chirp using the laser's internal prism sequence; simultaneously the energy coupled into the fiber was increased such that the amount of broadening, i.e., the spectral extent and Fourier limit of the output spectra, were kept constant.

Input pulse durations were inferred from the input chirp introduced by the prism displacement and also measured directly by autocorrelation. The pulse energies required for constant broadening are plotted in Fig. 2(a). Similarly to our earlier measurements (see Fig. 3 of [6] ), we again observe an increase in energy for longer pulses.

For compression, we used 12 reflections from a pair of double-angled chirped mirrors [ $\underline{8}]$ at $5^{\circ}$ and $22^{\circ}$ incidence per individual bounce. In summary, this provides a dispersion of about $-100 \mathrm{fs}^{2}$ for each double-bounce. This amount $\left(-1200 \mathrm{fs}^{2}\right)$ overcompensates the output chirp in all cases. For each setting of the input chirp, we hence inserted fused-silica glass blocks in order to optimize the compression. Final pulse durations of $\sim 17 \mathrm{fs}$ were obtained in all cases, measured by intensity autocorrelation. With the known dispersion of the glass

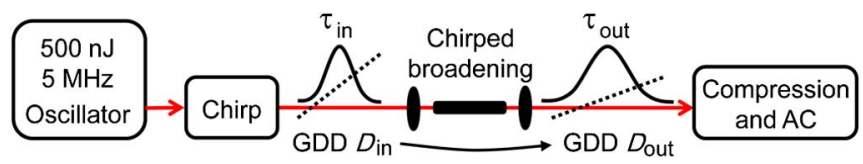

Fig. 1. Concept of our measurement of chirp transfer during broadening of stretched pulses in a large-mode-area fiber. Compression is made with chirped mirrors; input and output chirp (GDD) are determined by optimizing the pulse durations (AC, autocorrelation) with dispersive glass blocks. 

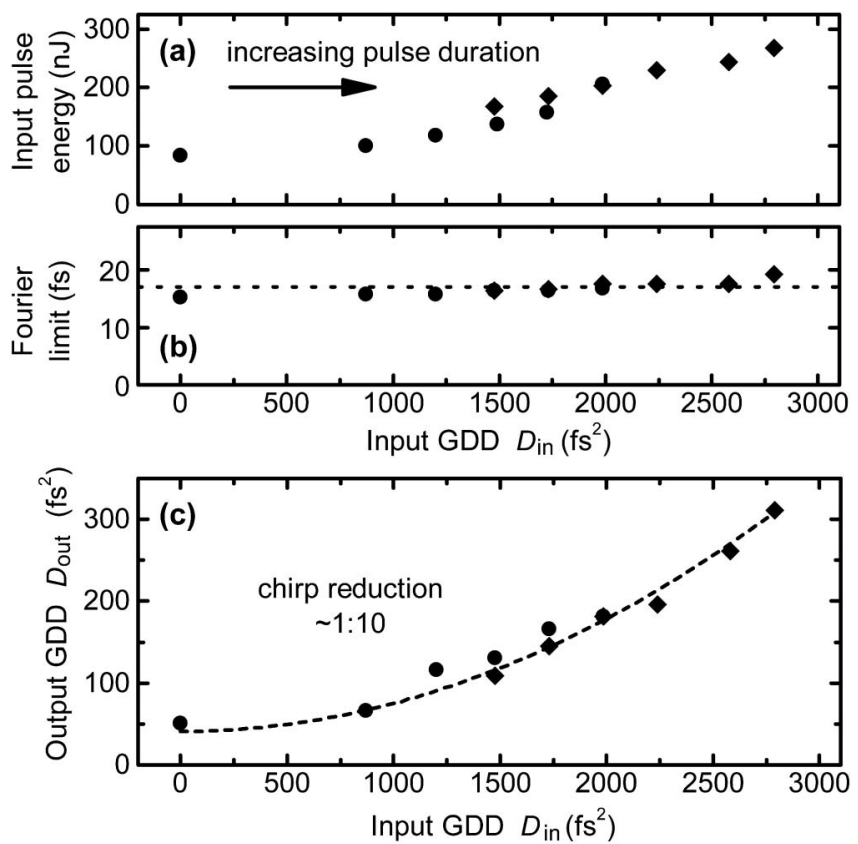

Fig. 2. Chirp transfer. (a) Measured input pulse energy required for maintaining a constant broadening, i.e., Fourier limit of $\sim 17 \mathrm{fs}$. (b) Fourier limit of the output spectrum for increasing input chirp and pulse energy. (c) Measured output chirp originating from the nonlinearity (i.e., with linear fiber dispersion subtracted) for different values of the input chirp. The dashed line is the result of a model based on considering self-phase modulation at an approximately constant pulse duration within the fiber. Dots and diamonds denote two different sets of measurements.

blocks and chirped mirror bounces, we inferred the chirp parameter of the output pulses. As a consequence of the short final pulse duration, autocorrelation was quite sensitive to chirp; we estimate an accuracy of about $\pm 30 \mathrm{fs}^{2}$ in this measurement. The fiber's linear dispersion (550 $\mathrm{fs}^{2}$ for our $15 \mathrm{~mm}$ long piece), the dispersion of the collimating lens and the dispersion of the air $\left(20 \mathrm{fs}^{2} / \mathrm{m}\right)$ between the fiber end and the autocorrelator were subtracted in order to solely study the input-tooutput chirp conversion due to nonlinearities.

Figures 2(b) and 2(c) show the results. Using the pulse energies plotted in Fig. 2(a), a Fourier limit of $\sim 17$ fs was obtained for all input chirps ranging from zero to $2800 \mathrm{fs}^{2}$ [see Fig. 2(b)]. The measured output chirps are plotted in Fig. 2(c). The output chirp is not larger than $350 \mathrm{fs}^{2}$ in any case. Compared to the range of input chirps, this represents a reduction by a factor of about 10 .

In order to understand this finding, we refrain from numerical fiber optics simulations $[\underline{1}, \underline{3}, \underline{9}]$ and aim for a predictive, analytical description.

We invoke here two hypotheses: first we assume that self-phase modulation (SPM) is the dominant mechanism for broadening; this is justified by the typical spectral shapes observed in all cases [6]. Second, we assume a constant pulse duration during the broadening process; dispersion is neglected. This model is justified by considering the order-of-magnitude of the characteristic lengths $L_{\mathrm{NL}}$ and $L_{D}$ of nonlinear broadening and of dispersion, respectively. With the peak intensity $I_{0} \approx 3.8$. $10^{11} \mathrm{~W} / \mathrm{cm}^{2}$ in the experiment, with a Fourier-limited pulse duration of $\tau_{0} \approx 55 \mathrm{fs}$, with fused-silica's nonlinear index of refraction of $n_{2} \approx 2.45 \cdot 10^{-16} \mathrm{~cm}^{2} / \mathrm{W}$ and with a fiber dispersion of $\beta_{2} \approx 36 \mathrm{fs}^{2} / \mathrm{mm}$, we obtain $L_{\mathrm{NL}}=$ $\lambda /\left(2 \pi n_{2} I_{0}\right) \approx 1.4 \mathrm{~mm}$ and $L_{D}=\tau_{0}^{2} / \beta_{2} \approx 85 \mathrm{~mm}$. Compared to the actual fiber length of $15 \mathrm{~mm}$, these values are well below and well above, respectively.

The chirp or temporal frequency modulation of a pulse is either characterized in the spectral domain by group delay dispersion (GDD) in units of $\mathrm{fs} / \mathrm{PHz}=\mathrm{fs}^{2}$, describing the group delay sweep over frequency, or in the temporal domain by the temporal chirp denoting the frequency sweep over time in $\mathrm{PHz} / \mathrm{fs}=\mathrm{fs}^{-2}$ units. For chirped Gaussian pulses, there is a relation between these two quantities, which simplifies to inverse proportionality if the stretched pulses are significantly longer $(>3)$ than the Fourier limit. In the experiment, we measure chirp in the spectral domain and obtain GDD values, but SPM and the chirp it causes are usually described in the temporal domain. Hence we approach the problem with a description in the time domain.

We denote the duration of the Fourier-limited input pulse by $\tau_{0}$ and the peak intensity by $I_{0}$, which is approximately constant for all combinations of input chirp and pulse energy [see Fig. 2(a)]. We describe the chirped input pulse by its center wavelength $\lambda_{0}$, center frequency $\omega_{0}$, GDD parameter $D_{\text {in }}\left(\right.$ in $\mathrm{fs}^{2}$ ), and duration $\tau_{\text {in }}(\mathrm{FWHM})$. The time-dependent intensity profile of a Gaussianshaped pulse can be written as

$$
I(t)=I_{0} \exp \left(-4(\ln 2) t^{2} / \tau_{\mathrm{in}}^{2}\right) \approx I_{0}\left(1-4(\ln 2) t^{2} / \tau_{\mathrm{in}}^{2}\right) .
$$

Here we approximate the Gaussian function with a parabola by Taylor expansion in order to simplify the derivatives required below. This is a good approximation of the peak region for a large variety of common pulse shapes in the femtosecond regime, not only for Gaussian-shaped pulses. With a GDD $D_{\text {in }}$, the pulse duration $\tau_{\text {in }}$ becomes

$$
\tau_{\text {in }}\left(D_{\text {in }}\right) \approx \tau_{0} \sqrt{1+(4 \ln 2)^{2} D_{\text {in }}^{2} / \tau_{0}^{4}} .
$$

For the output pulse, we assume a temporal profile with the same pulse duration as before and consider broadening by SPM. The time-dependent frequency $\omega(t)$ of a pulse after nonlinear broadening is

$$
\omega(t)=\omega_{0}-\frac{16(\ln 2)^{2} D_{\text {in }}}{\tau_{0}^{4}+(4 \ln 2)^{2}\left(D_{\text {in }}\right)^{2}} t-\frac{2 \pi}{\lambda_{0}} n_{2} L \frac{d I(t)}{d t},
$$

where $L=15 \mathrm{~mm}$ is the fiber's length and $n_{2} \approx$ $2.45 \cdot 10^{-16} \mathrm{~cm}^{2} / \mathrm{W}$ is the nonlinear index of refraction for fused silica. The B-integral has a value of $\sim 10$ for these parameters. The first term in Eq. (3) is the center frequency, the second term denotes the incoming pulse's temporal chirp (in $\mathrm{fs}^{-2}$ ), and the third term describes SPM induced by the time-dependent intensity profile. In our experiments at about $10^{11} \mathrm{~W} / \mathrm{cm}^{2}$, the nonlinear term is about 10 times larger than the original temporal chirp, which is therefore neglected: 


$$
\omega(t) \approx \omega_{0}-\frac{2 \pi}{\lambda_{0}} n_{2} L \frac{d I(t)}{d t} .
$$

Inserting the time-dependent intensity $I(t)$ given by Eq. (1), we obtain

$$
\omega(t) \approx \omega_{0}+\frac{16 \pi(\ln 2) n_{2} L I_{0}}{\lambda_{0} \tau\left(D_{\text {in }}\right)^{2}} t \approx \omega_{0}+t / D_{\text {out }} .
$$

This describes a variation of frequency with time, i.e., a chirp in the time domain. Here we made the approximation that the output temporal chirp is inversely proportional to the output GDD parameter $D_{\text {out }}$. This is generally only valid for pulses that are stretched significantly $(>3)$ with respect to the Fourier-limited pulse duration because only in this regime there is a direct relation between the definitions of the chirp parameter in the temporal or spectral domain, respectively. This assumption is valid in our experiments, as mentioned before. Finally, the output dispersion can be calculated from Eqs. (2) and (5):

$$
D_{\text {out }} \approx \frac{\lambda_{0} \tau\left(D_{\text {in }}\right)^{2}}{16 \pi(\ln 2) n_{2} I_{0} L} .
$$

This equation provides a prediction of the SPM-generated output GDD $D_{\text {out }}$ (neglecting linear fiber dispersion) in dependence of the input GDD $D_{\text {in }}$.

The results are plotted as the dashed line in Fig. 2(c). There was only one fit parameter applied for this curve, the peak intensity. We found $I_{0}=(4.6 \pm$ $0.1) \cdot 10^{11} \mathrm{~W} / \mathrm{cm}^{2}$, which is close to the experimental value of $3.8 \cdot 10^{11} \mathrm{~W} / \mathrm{cm}^{2}$ calculated from energy, pulse duration, and mode field diameter of about $20 \mu \mathrm{m}$. The agreement to the measured data is satisfactory. We find this remarkable because all parameters are fixed, and only a slight adjustment of the peak intensity was necessary to reproduce the experimental results.

We recall the two central approximations we made: (1) that other nonlinearities than SPM are negligible; (2) that the pulse duration is constant during the broadening process. The ability to explain the experimental results in this way indicates that these two features are indeed predominant in the regime that we study here.

All experiments reported so far were performed with the shortest fiber that provided a satisfactory broadening $(15 \mathrm{~mm})$. In a second experiment, we also measured the chirp transfer when using a $25 \mathrm{~mm}$ long fiber. About the same input energies were required for generating $17 \mathrm{fs}$ pulses as with the shorter fiber, but the output pulses were carrying a higher total output chirp, but at the same bandwidth. These two observations indicate that the significant part of the spectral broadening takes place in the first $10-15 \mathrm{~mm}$ of the fiber. Thereafter, the fiber's dispersion lengthens the pulses, suppressing further nonlinear broadening and producing longer pulses without further broadening. In this case, the above-derived simple model with constant duration loses its validity, and dispersion should be taken into account with a numerical split-step simulation $[1,3]$. We also tried shorter fibers than $15 \mathrm{~mm}$ in the experiment, but they did not perform well. In order to provide the best compromise between

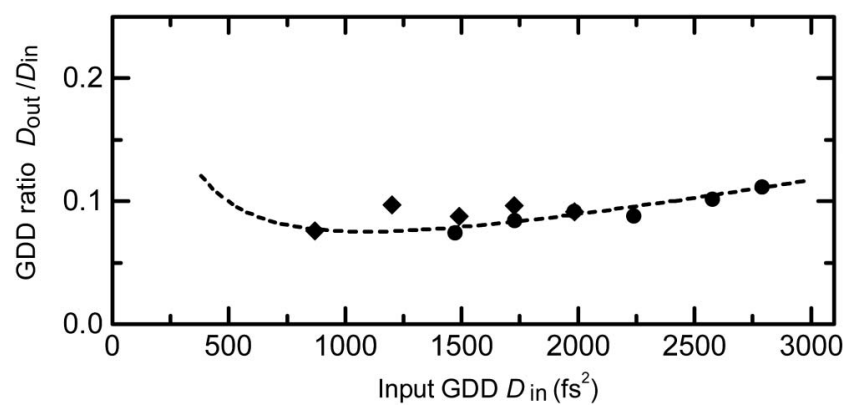

Fig. 3. Chirp reduction, plotted as the ratio of nonlinearityinduced output chirp to input chirp. The dashed line is a one-parameter fit of Eq. (6) and corresponds to the dashed line in Fig. 2(c).

broadening and chirp reduction, the fiber must have a certain optimum length, in our case $15 \mathrm{~mm}$.

Figure 3 shows the ratio $D_{\text {out }} / D_{\text {in }}$ of output chirp (caused by nonlinearities) to input chirp for the 15-mm fiber. In the measured range, the ratio is $\sim 1: 10$ and roughly constant.

Let us come back to the original question of whether chirped-pulse broadening may result in practically uncompressible output pulses. Here, the reported results indicate that the broadening of chirped input pulses instead of Fourier-limited pulses causes only a minor increase of the output dispersion that one must additionally compensate in the experiment. Compression of this additional dispersion is easily achievable with chirped mirrors. For example, the conventionally applied Fourier-limited input pulses require about five twofoldreflections from our chirped mirrors for compression $\left(-100 \mathrm{fs}^{2}\right.$ per double-bounce). For chirped pulses at the extreme points of the measurement (input GDD of around $2800 \mathrm{fs}^{2}$ ), less than four additional twofoldreflections are sufficient for the same compression at several-times-higher pulse energies. The throughput of this additional compression is $>92 \%$. This example shows that chirped-pulse broadening is a valuable approach in practice, thanks to the reduction of chirp reported here.

For increasingly longer input pulses, the ratio of chirp reduction becomes less favorable but still encouraging. The approximations made in deriving Eqs. (4) and (5) restrict the validity range of Eq. (6) to input pulse durations of $<300 \mathrm{fs}$. For longer pulses, a more exact calculation, still based on the physical principles (1) and (2) but without further simplifications, reveals that Eq. (6) provides an upper limit for the expected output dispersion. This allows us to predict the behavior of more intense pulses than available from our laser. For example, a chirped input pulse duration of $1000 \mathrm{fs}$ (input chirp of $\sim 20,000 \mathrm{fs}^{2}$ ) will probably allow to use $\sim 1.2 \mu \mathrm{J}$ for broadening to the $17 \mathrm{fs}$ regime. At an achievable efficiency of the fiber of $>80 \%$ [6] , we expect $1 \mu \mathrm{J}$ at the output. According to Eq. (6), which is invalid but providing an upper limit, the GDD after the fiber will be $\sim 13,000 \mathrm{fs}^{2}$; the more exact calculation yields $\sim 7700 \mathrm{fs}^{2}$, which is about $40 \%$ of the input GDD. Compression of $150 \mathrm{~nm}$ broad pulses in the $10,000 \mathrm{fs}^{2}$ regime is feasible using about 20 reflections from state-of-the-art chirped mirrors with stack thicknesses of $10 \mu \mathrm{m}$ and $-500 \mathrm{fs}^{2}$ per reflection [10]. 
Thanks to the mechanism of chirp reduction reported here, the generation of few-fs, $\mu \mathrm{J}$ pulses seems realistic using large-mode-area fibers in combination with chirping the input pulses. Hundreds of nJ were demonstrated experimentally ([6] and this work), and our concept can be combined with divided-pulse approaches [11]. Perceived applications range from ultrafast electron diffraction with single-electron pulses at $\mathrm{MHz}$ repetition rates $[12,13]$ to various experiments in ultrafast plasmonics performed with long-cavity oscillators [14]. Many other fields of research may also benefit from the mechanisms and perspectives reported here.

This work was supported by the Munich-Centre for Advanced Photonics, the Rudolf-Kaiser-Stiftung, and the European Research Council. P. D. acknowledges support from a Marie Curie Fellowship of the EU (project acronym "UPNEX"), and P. R. was supported by a postdoctoral grant of the Hungarian Academy of Sciences. We thank Thomas Ganz for some preliminary investigations [15].

\section{References}

1. P. Dombi, P. Antal, J. Fekete, R. Szipócs, and Z. Várallyay, Appl. Phys. B 88, 379 (2007).

2. P. Dombi and P. Antal, Laser Phys. Lett. 4, 538 (2007).
3. A. Fuerbach, C. Miese, W. Koehler, and M. Geissler, Opt. Express 17, 5905 (2009).

4. W. Koehler and G. Tempea, Proc. SPIE 7582, 75820B (2010).

5. J. Fekete, P. Rácz, and P. Dombi, Appl. Phys. B 111, 415 (2013).

6. T. Ganz, V. Pervak, A. Apolonski, and P. Baum, Opt. Lett. 36, 1107 (2011).

7. S. Naumov, A. Fernandez, R. Graf, P. Dombi, F. Krausz, and A. Apolonski, New J. Phys. 7, 216 (2005).

8. V. Pervak, I. Ahmad, M. K. Trubetskov, A. V. Tikhonravov, and F. Krausz, Opt. Express 17, 7943 (2009).

9. G. Agrawal, Nonlinear Fiber Optics, 5th ed. (Academic, 2013).

10. V. Pervak, V. Fedorov, Y. A. Pervak, and M. Trubetskov, Opt. Express 21, 18311 (2013).

11. A. Klenke, M. Kienel, T. Eidam, S. Hädrich, J. Limpert, and A. Tünnermann, Opt. Lett. 38, 4593 (2013).

12. P. Baum, Chem. Phys. 423, 55 (2013).

13. F. O. Kirchner, A. Gliserin, F. Krausz, and P. Baum, Nat. Photonics 8, 52 (2014).

14. P. Dombi, A. Hörl, P. Rácz, I. Márton, A. Trügler, J. R. Krenn, and U. Hohenester, Nano Lett. 13, 674 (2013).

15. T. Ganz, "Supercontinuum generation by chirped pulse compression for ultrafast spectroscopy and broadband near-field microscopy,” Ph.D. thesis (LMU München, 2011). 